\title{
Tatvan, Ahlat ve Adilcevaz Bölgelerinde Sahil Kumlarında Radon Aktivite Konsantrasyon Seviyelerinin Belirlenmesi
}

\author{
Şule Karatepe Çelik ${ }^{1}$, Muhammed Fatih Kuluöztürk ${ }^{2 *}$, Mahmut Doğru ${ }^{3}$ \\ ${ }^{1}$ Bitlis Eren Üniversitesi, Hizan Meslek Yüksekokulu, Bitlis, Türkiye (ORCID: 0000-0001-8125-2231) \\ ${ }^{2}$ Bitlis Eren Üniversitesi, Mühendislik-Mimarlık Fakültesi, Elektrik-Elektronik Mühendisliği, Bitlis, Türkiye (ORCID: 0000-0001-8581-2179) \\ ${ }^{3}$ Fırat Üniversitesi, Fen Fakültesi, Fizik Bölümü, Elazığ, Türkiye (ORCID: 0000-0002-0015-0629)
}

(Illk Geliş Tarihi 30 Ekim 2020 ve Kabul Tarihi 24 Aralık 2020)

(DOI: $10.31590 /$ ejosat.818568)

ATIF/REFERENCE: Karatepe Çelik, Ş., Kuluöztürk, M. F. \& Doğru, M. (2021). Tatvan, Ahlat ve Adilcevaz Bölgelerinde Sahil Kumlarında Radon Aktivite Konsantrasyon Seviyelerinin Belirlenmesi. European Journal of Science and Technology, (21), 90-94.

$\ddot{O} \mathbf{z}$

Radon gazı $\left({ }^{222} \mathrm{Rn}\right)$, hem doğal radyoaktivitenin büyük çoğunluğunu teşkil etmesi hem de insan sağlı̆̆ açısından akciğer kanserinin ikinci en önemli sebebi olması nedeniyle doğal radyoaktivite çalışmalarında önemli bir parametredir. Bu çalışmada, Van Gölü kıyı şeridinde yer alan Tatvan, Ahlat ve Adilcevaz beldelerinin sahil kumlarındaki radon gazı aktivite konsantrasyon seviyeleri belirlenmiştir. Nemrut ve Süphan sönmüş volkanlarının etkisiyle oluşan Van Gölünün sahil şeridinde önceden belirlenen 15 farklı noktadan sahil kumu örnekleri toplanmıştır. Toplanan örnekler kurutulup elendikten sonra CR-39 pasif katıhal iz detektörü (SSNTD - Solid State Nuclear Tracking Detector) ile birlikte bir kaba yerleştirilmiştir. 61 gün boyunca sahil kumlarından yayılan radon gazına maruz kalan detektörler çıkartılarak 4 buçuk saat boyunca $90^{\circ} \mathrm{C}$ 'de $6,25 \mathrm{M} \mathrm{NaOH}$ çözeltisi içinde kimyasal kazıma işlemine tabi tutulmuştur. Kazınmış detektörlerde RadoSYS otomatik sayım sistemi kullanılarak radon gazına ait izler sayılmıştır. İz yoğunluğu cinsinden alınan sayım sonuçları kullanılarak radon konsantrasyon seviyeleri belirlenmiş̧ir. En büyük ve en küçük konsantrasyon değerleri $459.42 \pm 7.41$ Bq.m${ }^{3}$ ve $105.34 \pm 3.09$ Bq. ${ }^{-3}$ olarak sırasıyla ADCZ-6 ve TTVN-12 örnek noktalarında elde edilmiştir. Ortalama aktivite değeri ise 193,30 Bq. $\mathrm{m}^{-3}$ olarak belirlenmiştir. Bölgesel olarak değerlendirildiğinde en yüksek aktivite değeri Tatvan'da ortalama $240,96 \mathrm{~Bq} \cdot \mathrm{m}^{-3}$, sonrasında Ahlat'ta 209,57 Bq. $\mathrm{m}^{-3}$ ve en düşük olarak Adilcevaz'da 129,36 Bq. $\mathrm{m}^{-3}$ olarak belirlenmiştir. Elde edilen aktivite değerleri kullanılarak Kriging metoduyla kontur dağılım haritası elde edilmiştir. Ayrıca örnekleme alanlarının yıllık etkin doz değerleri belirlenmiştir. En büyük ve en küçük doz değerleri sırasıyla $11,57 \mathrm{mSv} \cdot \mathrm{y}^{-1}$ ve $2,65 \mathrm{mSv} \cdot \mathrm{y}^{-1}$ olarak bulunmuştur. Elde edilen sonuçlar, Türkiye ve dünyanın diğer ülkelerinde farklı bölgelerdeki sahil kumlarının radon gazı seviyeleri ve limit değerler ile mukayese edilmiştir.

Anahtar Kelimeler: Radon Gazı, Sahil Kumları, Van Gölü.

\section{Determination of Radon Activity Concentration Levels of Beach Sands in Tatvan, Ahlat and Adilcevaz Regions}

\begin{abstract}
Radon gas $\left({ }^{222} \mathrm{Rn}\right)$ is an important parameter in natural radioactivity studies because it constitutes the majority of natural radioactivity and is the second most important cause of lung cancer in terms of human health. In this study, radon gas activity concentration levels were determined in beach sands of Tatvan, Ahlat and Adilcevaz regions which are on the shore of Lake Van. Beach sand samples were collected from 15 predetermined locations on the coastline of Lake Van, which was formed by the effect of Nemrut and Süphan extinct volcanoes. After the collected samples were dried and sieved, they were placed in a container with the CR-39 passive solid-state track detector (SSNTD - Solid State Nuclear Tracking Detector). The detectors exposed to the radon gas emitted from the beach sands for 61 days were removed and subjected to chemical etching at $90^{\circ} \mathrm{C}$ in $6.25 \mathrm{M} \mathrm{NaOH}$ solution for 4 and a half hours. Radon gas traces were counted on the etched detectors using the RadoSYS automatic counting system. Radon concentration levels were determined using the counting results in terms of trace density. The maximum and the minumum values were obtained as $459.42 \pm 7.41 \mathrm{~Bq} \cdot \mathrm{m}^{-3}$ and 105.34 $\pm 3.09 \mathrm{~Bq} . \mathrm{m}^{-3}$ in the ADCZ-6 sample and TTVN-12 sample, respectively. The average activity value was determined as $193.30 \mathrm{~Bq} . \mathrm{m}^{-}$ ${ }^{3}$. When evaluated regionally, the highest activity value was determined as an average of $240.96 \mathrm{~Bq} \cdot \mathrm{m}^{-3}$ in Tatvan, then $209.57 \mathrm{~Bq} \cdot \mathrm{m}^{-3}$
\end{abstract}

\footnotetext{
*Sorumlu Yazar: mfkuluozturk@beu.edu.tr
} 
in Ahlat and 129.36 Bq. $\mathrm{m}^{-3}$ in Adilcevaz. Using the activity values obtained, the contour distribution map was obtained by the Kriging method. Furthermore, annual effective dose values were determined for sand samples. The largest and smallest dose values were found to be $11.57 \mathrm{mSv} \cdot \mathrm{y}^{-1}$ and $2.65 \mathrm{mSv} \cdot \mathrm{y}^{-1}$, respectively. The obtained results were compared with limit values and radon gas levels of beach sands in the other different areas of Turkey and other countries.

Keywords: Radon Gas, Beach Sands, Lake Van.

\section{Giriş}

Radon gazı $\left({ }^{222} \mathrm{Rn}\right)$, doğadaki üç temel radyoaktif bozunum zincirinin tek gaz ara ürünü olan radyoaktif bir soy gazdır. Bolluk oranına göre sırasıyla yarılanma ömrü 3,82 gün olan ${ }^{222} \mathrm{Rn}$ (Radon) izotopu ${ }^{238} \mathrm{U}$ bozunum zincirinin, yarılanma ömrü 55,6 saniye olan ${ }^{220} \mathrm{Rn}$ (Thoron) izotopu ${ }^{232} \mathrm{Th}$ bozunum zincirinin ve yarılanma ömrü 3,96 saniye olan ${ }^{219} \mathrm{Rn}$ (Actinon) izotopu ${ }^{235} \mathrm{U}$ bozunum zincirinin üyesidir (Evans, R. D., 1968; Durrani, S. A. and İliç, R., 1997). Doğada en bol bulunan doğal radyoaktif bozunum zincirinin üyesi olması ve yarılanma süresinin diğer izotoplarına göre daha yüksek olmasından dolayı, radon gazı doğada daha yüksek konsantrasyonlarda bulunabilir ve radyoaktivite ölçümlerinde yaygın bir parametre olarak değerlendirilir.

Doğal radyoaktivitenin en büyük kısmını oluşturan radon gazı (yaklaşık \%55), insan sağlı̆̆ açısından radyoaktivite çalışmaları için önemli bir parametredir. İnsan solunum sistemine girdikten sonra, radon gazı hizlı bir alfa ve beta bozunum reaksiyon süreci geçirerek 138,37 günlük yarılanma ömrüne sahip toksik ${ }^{210} \mathrm{Po}$ izotopuna dönüșür. Akciğer kanserinin sigaradan sonra en önemli ikinci nedeni olan radon gazı, bu şekilde dolaylı olarak kansere neden olabilmektedir (WHO, 2014).

Kapalı yaşam alanlarında radon gazının insan sağlı̆̆ı açısından değerlendirilmesi konusunda birçok çalışma yapılmıştır. Örneğin; Çelebi vd. (2015) Türkiye konutlarında SSNTD (Katı Hal Nükleer İz Detektörü) kullanarak radon gazı ölçümleri yapmış ve 1 ile $1400 \mathrm{~Bq} \cdot \mathrm{m}^{-3}$ arasında değişen aritmetik ortalaması $81 \mathrm{~Bq} \cdot \mathrm{m}^{-3}$ olan değerler rapor etmişlerdir, Mihci vd. (2010) Sivas'ta mevsimlik iç ve dış radon konsantrasyonu ölçümleri yapmış ve iç ortam radon değerlerini yaz için 98 Bq.m ${ }^{3}$, kış için 89 Bq.m ${ }^{-3}$ olarak bildirmişlerdir, Kuluöztürk vd. (2018) yapı malzemesine bağlı olarak Ahlat'ta iç mekan radon konsantrasyonu ölçümleri yapmış ve 259,86 Bq.m ${ }^{-3}$ aritmetik ortalama ile 10.01 ile $2031.06 \mathrm{~Bq} . \mathrm{m}^{-3}$ arasında değişen değerler bildirmişlerdir, Kulalı vd. (2019) AlphaGuard aktif radon detektörü kullanarak bir üniversite kampüsündeki iç mekan radon gazı seviyelerini ölçmüş ve 7 ile $177 \mathrm{~Bq} \cdot \mathrm{m}^{-3}$ arasında değişen değerler bildirmişlerdir. Buna ek olarak radon gazıyla ilgili olarak bir çok dış mekan radyoaktivite çalışmaları da bulunmaktadır. Örneğin; Kulalı vd. (2017) AlphaGuard aktif radon detektörünü kullanarak meteorolojik parametrelerle ilişkili toprak gazı radonu üzerinde çalışmış ve 0.1 ila $160 \mathrm{kBq} \cdot \mathrm{m}^{-3}$ arasında değişen değerler rapor etmişlerdir, Yalım vd. (2006) AlphaGuard aktif radon detektörü kullanarak radon anomalileri ile depremler arasındaki ilişkiyi araştırmak için kuyu suyu üzerinde çalışmışlar ve genliği 2,6 ile 3,9 M arasında değişen depremlerle 0,79 ile 0,98 arasında korelasyonlar bulmuşlardır, Al-Fifi vd. (2012) sahil toprağ örneklerinde SSNTD'leri kullanarak radon konsantrasyonu ölçümleri yapmış ve 49.11 ile 151.79 Bq. ${ }^{-3}$ arasında değişen aritmetik ortalaması 88.18 Bq.m ${ }^{-3}$ olan değerler elde etmişlerdir.

$\mathrm{Bu}$ çalışmada, volkanik faaliyetler sonucu oluşan Van Gölü'nün Tatvan, Ahlat ve Adilcevaz kıylarında kıyı kum örneklerinde radon gazı aktivite konsantrasyonu CR-39 pasif iz detektörleri kullanılarak belirlenmiştir. Aktivite değerleri kullanılarak Kriging yöntemi ile kontur dağılım haritası elde edilmiştir. Aktivite değerlerine bağlı olarak yıllık efektif doz değerleri hesaplanmıştır.

\section{Materyal ve Metot}

\section{1. Örnek Toplama ve Hazırlama}

Van Gölü, Türkiye'nin en büyük gölüdür ve sönmüş Nemrut ve Süphan yanardağlarının volkanik faaliyetlerinin sonucu olarak meydana gelmiştir. Sahillerdeki kıyı kumlarının oluşumları, Van Gölü kıyılarında sönmüş Süphan ve Nemrut volkanlarının etkisiyle değişiklik göstermektedir. $\mathrm{Bu}$ nedenle, kıyı şeridinde mineralojik çeşitlilik vardır, bunun sonucunda radon gazı aktivite seviyelerinin de değişmesi beklenmektedir.

$\mathrm{Bu}$ çalışmada, radon gazı aktivite düzeylerinin belirlenmesi amaciyla Van Gölü'nün Bitlis ili Tatvan, Ahlat ve Adilcevaz ilçeleri kıyılarından sahil kumları toplanmıştır (Şekil 1). Yüzeyin $5-10 \mathrm{~cm}$ altında 15 farklı noktadan sahil kumu örnekleri toplanmıştır. Toplanan sahil kumu örnekleri Bitlis Eren Üniversitesi Bilim ve Teknoloji Uygulama ve Araştırma Merkezi Nükleer Fizik Laboratuvarı'na götürülerek, yaklaşık $110^{\circ} \mathrm{C}^{\prime} \mathrm{de}$ bir firında kurutulmuş ve daha sonra 60 mesh'lik bir elekle elenmiştir. Elenen numuneler, hava geçirmez difüzyon kabına 10 mm x 10 mm boyutlarında CR-39 detektörle birlikte yerleştirilmiş ve kapak sıkıca kapatılarak 61 gün pozlama için bekletilmiştir.

$\mathrm{Bu}$ sürenin sonunda difüzyon kaplarından çıkarılan detektörler, slaytlar üzerine yerleştirilmiş (slayt başına 12 dedektör) ve 4 buçuk saat boyunca $90^{\circ} \mathrm{C}^{\prime}$ de $6,25 \mathrm{M} \mathrm{NaOH}$ çözeltisi içinde kimyasal kazıma işlemine tabi tutulmuştur. Kimyasal kazıma işleminden sonra detektörler saf su ile yıkanıp kurutulduktan sonra detektör yüzeyindeki izler RadoSYS otomatik sayma sistemi ile sayılmıştır. Kimyasal kazıma ve otomatik sayım, RadoBATH ve RadoSYS sistemleri kullanılarak yapılmıştır (RadoSYS, 2011).

\subsection{Radon Gazı Aktivite Konsantrasyonlarının Belirlenmesi}

RadoSYS sistemi $\times 40$ büyütmeli optik mikroskoba sahiptir ve radon gazına ait izleri her bir detektör üzerinde $5,74 \mathrm{~mm}^{2}$ 'lik alanlara sahip 9 farklı noktada sayar. İstatistiksel hatayı azaltmak için her detektör $3 \mathrm{kez}$ sayılmıştır. Üretici tarafından verilen detektör kalibrasyon katsayısını içeren aşağıdaki denklem-1, sonuçları RadoSYS sistemi tarafindan verilen iz yoğunluğunu Bq. $\mathrm{m}^{-3}$ e dönüştürmek için kullanılır.

$$
\mathrm{C}_{\mathrm{Rn}}\left(\mathrm{Bq} \cdot \mathrm{m}^{-3}\right)=1000 \times \mathrm{TD} \times \mathrm{CF} / \mathrm{t}
$$

$\mathrm{Bu}$ denklemde $\mathrm{C}_{\mathrm{Rn}}, \mathrm{TD}, \mathrm{CF}$ ve $\mathrm{t}$ sirasiyla radon aktivite konsantrasyonunu (Bq. ${ }^{-3}$ ), iz yoğunluğunu (iz. $\mathrm{mm}^{-2}$ ), CR-39 detektörler için üretici firma tarafindan verilen kalibrasyon çarpanını $\left.\left(44,47 \text { h.kBq. }\left[\mathrm{m}^{3} \text {.(iz.mm } \mathrm{mm}^{-2}\right)\right]^{-1}\right)$ ve maruz kalma süresini (h) temsil etmektedir (RadoSYS, 2011). Radon gazı aktivite konsantrasyonlarından yola çıkarak yıllık etkin doz değeri, denklem-2 kullanılarak hesaplanmıştır.

$$
\mathrm{D}_{\mathrm{Rn}}\left(\mathrm{mSv} \cdot \mathrm{y}^{-1}\right)=\mathrm{C}_{\mathrm{Rn}} \times \mathrm{F} \times \mathrm{t} \times \mathrm{DCF}
$$


Bu denklemde $\mathrm{D}_{\mathrm{Rn}}, \mathrm{C}_{\mathrm{Rn}}, \mathrm{F}, \mathrm{t}$ ve DCF sirasıyla yıllık etkin dozu $\left(\mathrm{mSv} \cdot \mathrm{y}^{-1}\right)$, radon aktivite konsantrasyonunu (Bq. $\left.\mathrm{m}^{-3}\right)$, radon ve bozunum ürünleri arasındaki denge faktörünü $(0,4)$, ortalama yıllık harcanan zamanı (7000 h. $\left.\mathrm{y}^{-1}\right)$ ve aktivite-doz dönüşüm katsayısını (9 nSv.(Bq.h. $\left.\left.\mathrm{m}^{-3}\right)^{-1}\right)$ temsil etmektedir (UNSCEAR, 2000). Radon aktivite konsantrasyonu ve hesaplanan yıllık etkin

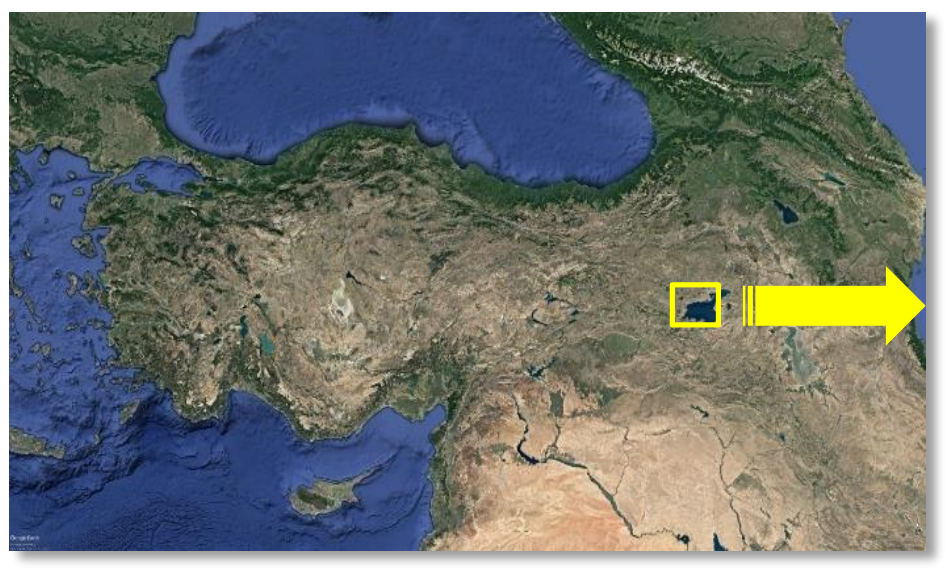

doz değerleri Tablo 1'de verilmiştir. Aktivite değerleri için Kriging metodu kullanılarak kontur dağılım haritası hazırlanmıştır (Şekil 2).

Şekil 1. Radon gazı aktivite belirleme noktaları.

\section{Araştırma Sonuçları ve Tartışma}

Elde edilen radon konsantrasyon değerleri ve yıllık etkin doz değerleri Tablo 1'de verilmiştir. Tablo 1'deki sonuçlar incelendiği zaman aktivite değerlerinin 105,34 ile 459,42 Bq. $\mathrm{m}^{-3}$ aralığında olduğu belirlenmiştir. Ayrıca ortalama değer 193,30 Bq. $\mathrm{m}^{-3}$ olarak bulunmuştur. Yıllık etkin doz değerleri 2,65 ile 11,57 mSv. $\mathrm{y}^{-1}$ arasında değişmektedir ve ortalama değeri ise 4,87 mSv. $\mathrm{y}^{-1}$ olarak hesaplanmıştır. Numune alanına göre ortalama radon aktivite konsantrasyon değeri ve yıllık etkin doz değeri Tablo 2'de ayrıca verilmiştir.

Tablo 2'deki sonuçlar incelendiğinde, radon aktivite konsantrasyon değeri Tatvan'da ortalama 240,96 Bq. $\mathrm{m}^{-3}$ ve yıllık doz değeri $6,07 \mathrm{mSv}^{-1} \mathrm{y}^{-1}$ olarak elde edildiği görülmektedir. Ahlat'ta ortalama radon aktivite konsantrasyon değeri 209,57 Bq. $\mathrm{m}^{-3}$ ve yıllık doz değeri 5,27 mSv. $\mathrm{y}^{-1}$ olarak bulunmuştur. Adilcevaz için radon konsantrasyon değeri ve yıllık doz oranı değeri $129,36 \mathrm{~Bq} \cdot \mathrm{m}^{-3}$ ve 3,25 mSv. $\mathrm{y}^{-1}$ olarak elde edilmiştir. $\mathrm{Bu}$ değerler göz önüne alındığında maksimum radon konsantrasyon değerinin Tatvan bölgesinden elde edildiği ve minimumum değerin ise Adilcevaz bölgesinde olduğu belirlenmiştir. Ahlat bölgesindeki sonuçların ise Tatvan bölgesinde elde edilen sonuçlara yakın olduğu görülmektedir.

\section{Sonuç}

Bu çalışmada, Van Gölü kıyısında bulunan Tatvan, Ahlat ve Adilcevaz bölgelerindeki sahil kumlarından toplanan numunelerin radon aktivite konsantrasyonu belirlenmiştir. Radon konsantrasyon değeri belirlenmesi için CR-39 pasif iz detektörü kullanılmıştır. Numuneler belirlenen konumlardan toplanmış ve numune hazırlama süreci ile analizler Bitlis Eren Üniversitesi Bilim ve Teknoloji ve Araştırma Merkezi Nükleer Fizik laboratuvarında yapılmıştır. Elde edilen sonuçlar değerlendirildiğinde radon gazı aktivite konsantrasyonunun ortalama değerinin 193,30 Bq.m-3 olduğu görülmektedir. Bu değer, Türkiye Atom Enerjisi Kurumu tarafından belirlenen izin verilen kapalı alan radon gazı aktivite konsantrasyonu 400 Bq.m3 değerinin altındadır (TAEK, 2000). Bu sınır değerin sadece Tatvan ile Ahlat arasındaki iki noktada (TTVN-05 ve AHLT-07) aşıldığı görülmektedir. Aktivite konsantrasyon değerlerinin yüksek olduğu bu bölge, sönmüş Nemrut yanardağının yamaçlarında yer almaktadır. Kuluöztürk vd. (Kuluöztürk vd. 2018), Ahlat'taki evlerde CR-39 kullanarak radon gazı aktivite konsantrasyonunun ortalama aktivite değerini 259,86 Bq.m-3 ve ortalama doz değeri $6,55 \mathrm{mSv} . \mathrm{y}-1$ olarak belirlemişlerdir. $\mathrm{Bu}$ çalışmadan elde edilen sonuçların 2018 yılında elde edilen sonuçlarla uyumlu olduğu görülmüştür. Mevcut çalışmaya benzer şekilde, Al-Fifi vd. (Al-Fifi vd. 2012) CR-39 detektörleri kullanarak Jazan limanından alınan sahil kumlarında radon gazı aktivite konsantrasyonlarını belirlemiş ve aktivite değerlerinin ortalama 88,18 Bq.m-3 ve 49,11 ile 151.79 Bq.m-3 arasında değiştiğini bildirmişlerdir. $\mathrm{Bu}$ değer, bu çalışmada elde edilen ortalama değerin yaklaşık yarısına karşılık gelmektedir. $\mathrm{Bu}$ çalışma alanlarının jeolojik farklılıklarından kaynaklanmaktadır.

\section{Teșekkür}

$\mathrm{Bu}$ çalışma, Bitlis Eren Üniversitesi Bilimsel Araştırma Projeleri Destek Birimi tarafından desteklenmiştir (Proje No: 2018.09). 
European Journal of Science and Technology

Tablo 1. Radon aktivite konsantrasyon ve yıllık efektif doz oranı değerleri

\begin{tabular}{c|c|c|c|c}
\hline Numune No & Enlem & Boylam & $\left.\mathbf{C}_{\mathbf{R n}} \mathbf{( B q . \mathbf { m } ^ { - 3 }}\right)$ & $\mathbf{D}_{\mathbf{R n}}\left(\mathbf{m S v . \mathbf { y } ^ { - 1 }}\right)$ \\
\hline TTVN-01 & 38,481397 & 42,307344 & $185,40 \pm 0,60$ & 4,67 \\
\hline TTVN-02 & 38,528620 & 42,313645 & $205,71 \pm 0,61$ & 5,18 \\
\hline TTVN-03 & 38,527748 & 42,316802 & $173,45 \pm 1,54$ & 4,37 \\
\hline TTVN-04 & 38,549531 & 42,356442 & $180,82 \pm 8,02$ & 4,55 \\
\hline TTVN-05 & 38,585319 & 42,389500 & $459,42 \pm 7,41$ & 11,57 \\
\hline AHLT-06 & 38,617119 & 42,404628 & $203,32 \pm 0,30$ & 5,12 \\
\hline AHLT-07 & 38,627794 & 42,457897 & $447,47 \pm 4,94$ & 11,27 \\
\hline AHLT-08 & 38,717861 & 42,430653 & $127,84 \pm 4,63$ & 3,22 \\
\hline AHLT-09 & 38,754756 & 42,510156 & $128,44 \pm 6,17$ & 3,23 \\
\hline AHLT-10 & 38,756142 & 42,518236 & $140,79 \pm 2,16$ & 3,54 \\
\hline ADCZ-11 & 38,780250 & 42,607869 & $182,61 \pm 1,50$ & 4,60 \\
\hline ADCZ-12 & 38,797731 & 42,736194 & $133,62 \pm 8,95$ & 3,36 \\
\hline ADCZ-13 & 38,794808 & 42,747939 & $105,34 \pm 3,09$ & 2,65 \\
\hline ADCZ-14 & 38,784856 & 42,842944 & $118,09 \pm 0,61$ & 2,97 \\
\hline ADCZ-15 & 38,787064 & 42,845142 & $107,14 \pm 1,24$ & 2,69 \\
\hline Min. & & 105,34 & 2,65 \\
\hline Mak. & & 459,42 & 11,57 \\
\hline Ort. & & 193,30 & 4,87 \\
\hline
\end{tabular}

Tablo 2. Bölgesel radon gazı aktivite konsantrasyonu ve yıllık efektif doz oranı değerleri.

\begin{tabular}{|c|c|c|c|c|}
\hline \multirow{2}{*}{ Çalışma Alanı } & \multicolumn{2}{|c|}{$C_{R n}\left(B q \cdot m^{-3}\right)$} & \multicolumn{2}{|c|}{$D_{R n}\left(m S v . y^{-1}\right)$} \\
\hline & Değişim Aralığı & Ortalama & Değişim Aralığı & Ortalama \\
\hline Tatvan & $180,82-459,42$ & 240,96 & $4,37-11,57$ & 6,07 \\
\hline Ahlat & $127,84-447,47$ & 209,57 & $3,22-11,27$ & 5,27 \\
\hline Adilcevaz & $105,34-182,61$ & 129,36 & $2,65-4,60$ & 3,25 \\
\hline \multicolumn{2}{|l|}{ TAEK } & 400 & & \\
\hline \multicolumn{2}{|l|}{ Al-Fifi } & 88,18 & & \\
\hline \multicolumn{2}{|l|}{ Kuluöztürk } & 259,86 & & \\
\hline
\end{tabular}

38.8 


\section{Kaynakça}

Durrani, S. A. and İliç, R., (1997). Radon Measurements by Etched Track Detectors: Applications in Radiation Protection. Earth Sciences and the Environment, World Scientific, New Jersey, U.S.A., 387p.

Evans, R. D. (1968). Engineers' Guide to The Elementary Behavior of Radon Daughters. Health Physics, Vol. 38, pp. 1173-1197. DOI: 10.1097/00004032-196908000-00006.

World Health Organisation (WHO) (2014). World Cancer Report. WHO Press.

Çelebi N., Ataksor B., Taskın H. and Albayrak Bingoldag N. (2015). Indoor radon measurements in Turkey dwellings. Radiation Protection Dosimetry, vol. 167, no. 4, pp. 626632. doi:10.1093/rpd/ncu329.

Mihci M, Buyuksarac A, Aydemir A, Celebi N. (2010). Indoor and outdoor Radon concentration measurements in Sivas, Turkey, in comparison with geological setting. Journal of Environmental Radioactivity, 101:952-957. https ://doi.org/10.1016/j.jenvr ad.2010.06.013.

Kuluöztürk M.F., Büyüksaraç A., Özbey F., Yalçin S. and Doğru M. (2018). Determination of indoor radon gas levels in some buildings constructed with Ahlat stone in Ahlat/Bitlis. International Journal of Environmental Science and Technology, Volume 16, Issue 9, pp 50335038. https://doi.org/10.1007/s13762-018-1692-0.

Kulalı F., Günay O. and Aközcan S. (2019). Determination of indoor radon levels at campuses of Üsküdar and Okan Universities. International Journal of Environmental Science and Technology (16) 5281-5284. https://doi.org/10.1007/s13762-019-02369-5.

Kulalı F., Akkurt I. and Özgür N. (2017). The Effect of Meteorological Parameters on Radon Concentration in Soil Gas. Acta Physica Polonica A, Vol. 132, No. 3-II, 9991001. DOI: 10.12693/APhysPolA.132.999.

Günay, O., Saç, M. M., İçhedef, M., Taşköprü, C. (2018). Soil gas radon concentrations along the Ganos Fault (GF). Arabian Journal of Geosciences, 11(9), 213.

Günay, O., Aközcan, S., Kulalı, F. (2018). Bina İçi Radon Konsantrasyonlarının Belirlenmesi. Avrupa Bilim ve Teknoloji Dergisi, (13), 91-97.

Günay, O., Aközcan, S., \& Kulalı, F. (2019). Measurement of indoor radon concentration and annual effective dose estimation for a university campus in Istanbul. Arabian Journal of Geosciences, 12(5), 171.

Yalım H.A., Sandıkcıoğlu A., Ertuğrul O. and Yıldız A. (2012). Determination of the relationship between radon anomalies and earthquakes in well waters on the AkşehirSimav Fault System in Afyonkarahisar province, Turkey. Journal of Environmental Radioactivity, 110, 7-12. DOI:10.1016/j.jenvrad.2012.01.015.

Al-Fifi Z., El-Araby E.H., Elhaes H. (2012). Monitoring of Radon Concentrations in Jazan Beach Soil. Journal of Applied Sciences Research, 8(2): 823-827.

RadoSYS (2011). RadoSYS Radometer 2000 User Manual. Hungary.
UNSCEAR (United Nations Scientific Committee on the Effects of Atomic Radiation) (2000). Sources and Effects of Ionizing Radiations. UN, New York.

TAEK (Turkish Atomic Energy Authority) (2000). Regulations for the radiation safety. Official Journal, Date/Number: 24.03.2000 / 23999. 